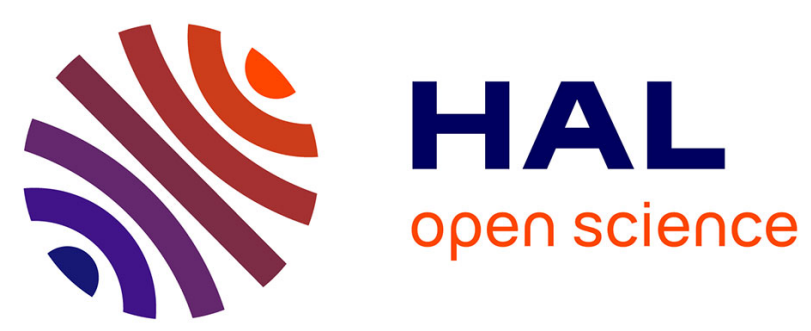

\title{
In Silico Voting Experiments
}

Jean-François Laslier

\section{To cite this version:}

Jean-François Laslier. In Silico Voting Experiments. 2009. hal-00390376

\section{HAL Id: hal-00390376 \\ https://hal.science/hal-00390376}

Preprint submitted on 2 Jun 2009

HAL is a multi-disciplinary open access archive for the deposit and dissemination of scientific research documents, whether they are published or not. The documents may come from teaching and research institutions in France or abroad, or from public or private research centers.
L'archive ouverte pluridisciplinaire HAL, est destinée au dépôt et à la diffusion de documents scientifiques de niveau recherche, publiés ou non, émanant des établissements d'enseignement et de recherche français ou étrangers, des laboratoires publics ou privés. 


\title{
ECOLE POLYTECHNIQUE
}

CENTRE NATIONAL DE LA RECHERCHE SCIENTIFIQUE

\author{
In Silico Voting Experiments
}

Jean-François LASLIER

May 2009

Cahier n $^{\circ}$ 2009-18

\section{DEPARTEMENT D'ECONOMIE}

Route de Saclay

91128 PALAISEAU CEDEX

(33) 169333033

http://www.enseignement.polytechnique.fr/economie/

mailto:chantal.poujouly@polytechnique.edu 


\title{
In Silico Voting Experiments
}

\author{
Jean-François LASLIER \\ Department of Economics, École Polytechnique \\ Jean-Francois.Laslier@polytechnique.edu
}

May 14, 2009

\begin{abstract}
This paper presents computer simulations of voting rules: Plurality rule, Approval voting and the Copeland and Borda rules, with voters voting sincerly or strategically. Different ways of generating random preference profiles are introduced: Rousseauist cultures are suitable for common interest project assessment; Impartial cultures are standard in Social Choice Theory; Distributive cultures and Spatial Euclidean ones are standard in Political Science.
\end{abstract}

\section{Introduction}

This chapter is devoted to computation-based simulations of voting. To perform such a simulation requires two things. On one hand, one has to specify the economic environment, that is the number of voters, the number of alternatives, and the voter preferences (or tastes, values, utilities, opinions...) over the alternatives. On the other hand, one has to specify the decision process, that is the voting rule and how a voter decides to place in the urn one ballot rather than another, given her preferences and any other relevant information.

The random generation of a profile of voter preferences is usually called a culture. For instance choosing $n$ individual preferences uniformly and independently among the $K$ ! linear orderings of $K$ alternatives is called the impartial culture of size $(n, K)$. Several different cultures will be studied in this chapter. Different cultures may be relevant to model different real-life voting situations: juries, project assesment, commeetee decisons, political elections,.... The individuals will often be called "voters" and will receive female pronouns, and I shalll sometime refer to alternatives as "candidates" and use male pronoums in that case. 
The second ingredient is the voting scheme: voter behavior under a voting rule. For instance, under the familiar Plurality rule, a voter can vote for her preferred candidate, not taking into account what she knows about the relative chances of winning of the various candidates. This behavior called sincere voting is well defined under Plurality rule (up to indiferences). Different voting schemes, sometime including strategic considerations will be studied. I will restrict attention to some practical voting rules which, up to unavoidable ties, select a winner: Plurality, Borda, Copeland and Approval voting. I will not compute choice correspondences like the Uncovered set, the Essential set or the Yolk which are set-valued in practice.

Section 2 introduces the cultures this chapter deals with: (1) Common interest cultures, in the tradition of Rousseau and Condorcet; (2) Impartial culture, often considered by mathematicians and social choice theorists; (3) Distributive cultures, suitable for the study of the "Divide a dollar" problem; (4) Spatial Euclidean cultures, often met in Theoretical Politics. Section 3 introduces the voting rules and voter behavior under scrutinity. Section 4 contains the results and Section 5 concludes.

\section{Cultures}

\subsection{Rousseauist cultures}

In this section I model individual preferences that may differ because of mistakes individuals make when forming their opinion about a pre-existing truth. This the typical approach of Condorcet: differences of opinions are due to differential information or to mistakes with respect to some underlying truth that collective decision-making can discover. Such a conceptual framework is called "project assessment' by Nurmi and Salonen (2008). It is the framework of the original "Condorcet Jury theorem" (Condorcet 1785) whose philosophy follows from Rousseau's ideal (Rousseau 1762) of a "general will." I therefore refer to Rousseau and call such cultures Rousseauist cultures.

To model the notion of individual mistakes I suppose that there exists an underlying true ranking of the alternatives, say

$$
1 \succ 2 \succ \ldots \succ K
$$

and that each voter is correct with probability $p\left(k, k^{\prime}\right)$ when comparing $k$ and $k^{\prime}$. I suppose that these mistakes are independent from one voter to another. The alternative number one, the "true" best alternative will be called the Rousseau alternative. 
I use the two-parameter formulation of Truchon and Drissi-Bakhkhat (2004) and Truchon (2008) which states that for some $\alpha \geq 0$ and $\beta \geq 0$, for all $k<k^{\prime}$ :

$$
p\left(k, k^{\prime}\right)=\frac{e^{\alpha+\beta\left(k^{\prime}-k-1\right)}}{1+e^{\alpha+\beta\left(k^{\prime}-k-1\right)}} .
$$

Note that for $k<k \prime$ :

$$
1 / 2 \leq p\left(k, k^{\prime}\right)<1 .
$$

I suppose that each voter gives one consistent opinion on each pair: if individual $i$ reports that she prefers $k$ to $k^{\prime}$, she does not reports that she prefers $k^{\prime}$ to $k$. But I do not require the individual to be consistent across pairs: If $i$ reports that she prefers $k$ to $k \prime$ and $k^{\prime}$ to $k^{\prime \prime}$, she may reports that she prefers $k^{\prime \prime}$ to $k$ (of course this implies that she has made at least one mistake). This framework is called the Rousseauist culture of size $(n, K)$ and parameters $(\alpha, \beta)$.

The information reported by each individual is therefore a tournament (complete and asymetric binary relation) over the set of alternatives The most likely reported tournament is the true ranking of the alternatives.

For $\beta=0$ the probability of a mistake does not depend on the ranks of the alternatives, as in Young (1988). The above model with $\beta \geq 0$ is more flexible and it seems reasonable to postulate that the probability of an error is larger when comparing two alternatives closer one to each other in the true underlying ranking. When $\beta$ or $\alpha$ is large, $p\left(k, k^{\prime}\right)$ tends to one, which means that the voter's expertise is very good.

With a number $n$ of voters, the individual preferences over pairs of alternatives define a vote matrix $M$ of size $K \times K$, in which the entry $m_{k, k^{\prime}}$ is the number of voters who prefer $k$ to $k^{\prime}$, with $m_{k, k^{\prime}}+m_{k^{\prime}, k}=n$. For convenience one can define on the diagonal $m_{k, k}=n / 2$.

A Condorcet winner can be defined in this framework: it is an alternative $k$ such that $m_{k, k^{\prime}} \geq n / 2$ for all $k^{\prime}$. In the simulations I will always take $n$ odd, so that there is no need to distinguish strict from large inequalities in this definition. As usual a Condorcet winner needs not to exist but, if it exists, it is unique.

Any rule based on pairwise comparisons may be computed in this framework. For instance the Borda rule may be applied, even if some individual preferences are not transitive: as it is well known, the Borda score of an alternative $k$ is the sum:

$$
b s(k)=\sum_{k^{\prime}=1}^{K} m_{k, k^{\prime}}
$$


and a Borda winner is and alternative with highest Borda score. ${ }^{1}$

\subsection{Impartial culture}

The Impartial culture for $n$ voters and $K$ alternatives is obtained by chosing each individual preference at random uniformly among the $K$ ! linear orderings of the alternatives, and independently of the preferences of the other voters. One thus obtains the uniform probability distribution over the set of profiles of linear orders. In this culture, there is a complete symmetry among alternatives: learning something on the relative ranking by some indivudals of some alternatives gives no information on the other individuals or alternatives.

This mathematically simple culture has been widely studied in the social choice literature. For instance it is known that the probability in this culture of the existence of a Condorcet winner is growing with the number of voters and the number of alternatives. See Gehrlein and Fishburn (1979), Gehrlein (1997).

\subsection{Distributive cultures}

Distributive cultures describe societies of complete antagonism. They are generated as follows. One unit of a divisible good (a "cake") has to be shared among $n$ individuals. Each individuals wants her share to be as large as possible, and does not care about the other shares. The set of alternatives is here infinite, it is the $n$-simplex:

$$
\Delta_{n}=\left\{x \in R^{n}: 0 \leq x_{i}, \sum_{i=1}^{n} x_{i}=1\right\} .
$$

Theoretical models of redistributive politics (Lindbeck and Weibull 1987, Myerson 1993, Lizzeri 1999, Laslier 2002, Laslier and Picard 2002) use economic environments which are identical or related to this set of alternatives. There is no obvious "natural" probability distribution over this set, and I will use several such distributions.

\subsubsection{Consensual redistributive culture}

Here, I use the projection on $\Delta_{n}$ of the uniform distribution on the cube $[0,1]^{n}$. This distribution is most easy to simulate: one chooses at random

\footnotetext{
${ }^{1}$ The reader will easily make the connection with the other, equivalent, definition of the Borda ruel using sum of ranks.
} 
(independently and uniformly between 0 and 1) numbers $y_{i}^{k}$ for $i=1, \ldots, n$ and $k=1, \ldots, K$ and then computes

$$
x_{i}^{k}=\frac{y_{i}^{k}}{\sum_{j=1}^{n} y_{j}^{k}} .
$$

Ties can be neglected so that this process defines a random profile of linear orders on $K$ alternatives for $n$ voters by setting

$$
x^{k} P_{i} x^{k^{\prime}} \Longleftrightarrow x_{i}^{k}>x_{i}^{k^{\prime}} .
$$

Note that even if this culture describes a situation of complete antagonism, it is not clear wether alternatives in this culture are typically very unequal distribution or close to the equal split. A first observation is that, when the number $n$ of individuals is large, the probability distribution on $\Delta_{n}$ tends to concentrate around the point of equal division $(1 / n, 1 / n, \ldots, 1 / n)$. This can be seen in the simulations by computing the standard deviation

$$
d(x)=\sqrt{\frac{1}{n} \sum_{i=1}^{n}\left(x_{i}-\frac{1}{n}\right)^{2}}
$$

which is also the Euclidean distance between the point $x$ and the equal division $(1 / n, 1 / n, \ldots, 1 / n)$. This random quantity tends, in expectation, to 0 when $n$ is large. (See the Appendix.) Therefore this culture may be seen as consensual, or even egalitarian, because it describes a society who tends to imagine solutions to the pure redistribution problem which are close (according to the Euclidean distance) to the perfectly egalitarian one.

But inequality is usually measured not by the standard deviation but by specific indices such as the Gini index of inequality. Let $u_{i}$ be the share of the $i$-th poorest individual and let $v_{i}$ be the total share of the $i$-th poorest individuals:

$$
\begin{aligned}
u_{1} & \leq u_{2} \leq \ldots \leq u_{n} \\
v_{i} & =\sum_{j \leq i} u_{j} .
\end{aligned}
$$

In the case where the shares are all identical, $u_{i}=1 / n$ and $v_{i}=i / n$. The increasing numbers $v_{i}$, for $i=1, \ldots, n$, define the concentration of the distribution and one can measure how concentrated (or "unequal") the distribution is by the Gini index

$$
\operatorname{gini}(x)=\frac{2}{n} \sum_{i=1}^{n}\left(\frac{i}{n}-v_{i}\right) .
$$




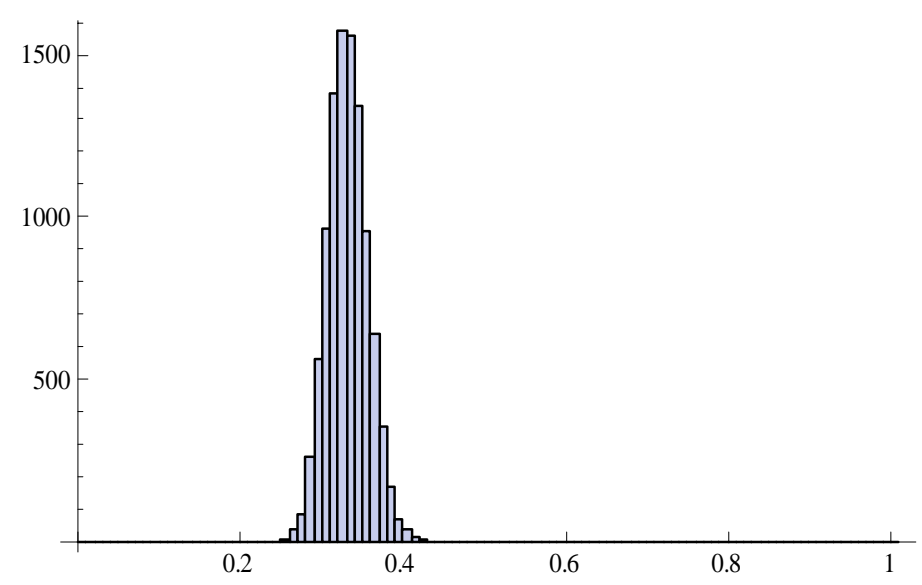

Figure 1: Consensual distributive culture, 99 individuals: histogram for the Gini index.

\begin{tabular}{|c|c|c|c|c|c|c|}
\hline$n$ & 3 & 5 & 11 & 49 & 99 & 999 \\
\hline Standard deviation & .16 & .10 & .05 & .01 & .006 & .00006 \\
\hline Gini index & .25 & .28 & .31 & .33 & .33 & .33 \\
\hline
\end{tabular}

Table 1: Consensual distributive culture: Standard deviation and Gini index depending on the number of voters

This coefficient ${ }^{2}$ is between 0 and 1 .

If one measures inequality by the Gini index of inequality, one reaches a different conclusion: When $n$ is large the expected value of the Gini index tends to $1 / 3$. This is a non-degenerated value and, arguably, a relatively small one. For these reasons I chose to call this culture the consensual distributive culture. Picture 1 shows the distribution of the values taken by this index in a society of 99 individuals.

Empirical averages for the standard deviation and the Gini index are provided in Table 1

\subsubsection{Inegalitarian distributive cultures}

In order to introduce these cultures, consider first that the scale of shares obtained by the individuals is fixed and linear: the poorest individual gets

\footnotetext{
${ }^{2}$ I use here a slightly simplified version of the Gini index. For a discrete distribution, the exact formula is $\frac{2}{n} \sum_{i=1}^{n}\left(\frac{i-1 / 2}{n}-\frac{v_{i}+v_{i-1}}{2}\right)$.
} 


\begin{tabular}{|c|c|c|c|c|c|c|c|c|}
\hline$e$ & 1 & 1.2 & 1.5 & 2 & 3 & 4 & 5 & 6 \\
\hline Gini & 0 & .1 & .2 & .33 & .5 & .6 & .66 & .7 \\
\hline
\end{tabular}

Table 2: Gini coefficient depending on the parameter $e$

some amount $t$, the second poorest gets $2 t$, the third gets $3 t$, and so on up to the richest individual who gets $n t$. Then the total amount is

$$
(1+2+\ldots+n) t=\frac{n(n+1)}{2} t
$$

and, in order that the individual shares add to 1 , one sets $t=\frac{2}{n(n+1)}$.

A possible alternative is the assignments of these shares to individuals. There are thus $n$ ! different possible alternatives. This defines a culture if these fixed shares are randomly assigned to the individuals. One picks at random $K$ of these redistributions, independently and uniformly to define the linear-inegalitarian distributive culture of size $(n, K)$. Note that in this culture, unlike the previous case the amount of inequality is the same in any alternative.

Starting from this idea, one can generalize it and define more or less egalitarian redistributive cultures by changing the linear scale $(t, 2 t, \ldots, n t)$ to a non linear one; then one can measure inequality by the usual Gini index. This is what I will do now.

To generate preference profiles, consider a one-parameter familly of concentration curves

$$
x \mapsto x^{e}
$$

for $e \geq 1$. In a society of $n$ individuals with such concentration, the $i$ poorest individuals together get

$$
v_{i}=\left(\frac{i}{n}\right)^{e}
$$

which means that the poorest individual has $u_{1}=\left(\frac{1}{n}\right)^{e}$, the second poorest has $u_{2}=\left(\frac{2}{n}\right)^{e}-\left(\frac{1}{n}\right)^{e}$, and so on up to the richest individual who has $u_{n}=1-\left(\frac{n-1}{n}\right)^{e}$.

The inequality of such a redistribution depends on $e$ (it is approximately independent of $n$ ). The Gini coefficients are given in Table 2.

Typical real values for the Gini index of income distributions at the national level are .25 in Sweden, .33 in France, .45 in the US, .59 in Brasil and more than .7 in some African countries.

I define the $\left(e_{\min }, e_{\max }\right)$-inegalitarian distributive culture of size $(n, K)$ as follows. For each $k$ independently (with $1 \leq k \leq K$ ) a parameter $e_{k}$ is 
picked at random uniformly on the interval $\left[e_{\min }, e_{\max }\right]$, then alternative $k$ is chosen according to the concentration parameter $e_{k}$, by assigning randomly the specified shares to the individuals.

As mentionned above, distributive cultures are interesting models of Politics: an alternative is a political platform that offers some amount to the different voters. One problem with this approach is that it is not reasonable to imagine that actual poltical platforms can target individual voters one by one. But certainly they can target social groups. To this respect, remark that the distributive culture introduced in this section well describes a situation where there are not $n$ individuals but $n$ groups of individuals, the groups being of equal size. Each of the $K$ candidates then choses to favor more or less the various groups. For this reason I find pertinent, as a model of large politics, to consider inegalitarian distributive cultures for relatively small values of the parameter $n$.

\subsection{Spatial cultures}

These cultures stem from the spatial theory of voting. In the Euclidean space $\mathbb{R}^{d}$ with $d$ dimensions, each voter $i$ has a bliss point $\omega_{i}$ and a utility function defined on $\mathbb{R}^{d}$ which is decreasing with the distance to $\omega_{i}$ :

$$
u_{i}(x)=-\left\|x-\omega_{i}\right\|
$$

An alternative is a point in $\mathbb{R}^{d}$ and a culture is defined by the number of dimensions $d$ and the probability distributions for $n$ bliss points and $K$ alternatives.

\subsubsection{Uni-dimensional spatial culture}

As it is well known, for $d=1$, a preference profile generated by the above single-peaked utility functions always has a Condorcet winner, which is the availlable alternative closest to the median bliss point (see for instance Austen-Smith and Banks (1999) for details). This culture is an easy way to generate profiles with this property.

\subsubsection{Multi-dimensional cultures}

With more than one, it may be the case or not. From the theory (McKelvey 1986), one may expect that (as soon as there are more than three voters) if the number of candidate is large, the probability that one of them is a Condorcet winner becomes very small; but this clearly cannot be true in general 
and indeed depends on the probability distributions on bliss points and alternatives. For simplicity I will use only uniform distributions over bounded boxes (intervals, rectangles,...), with the same probability distributions for bliss points and for alternatives ${ }^{3}$.

If the box is very thin, then the profiles become similar to one-dimensional profiles. If the rectangle or the box is far from degenerated, the uniform choice of bliss points and alternatives may lead to think that, if the numbers of voters and alternatives are large, the profile can be qualitatively described by a model with a continum of voters and alternatives uniformly distributed. This convergence question is a delicate theoretical issue. McKelvey and Tovey (2008) study the convergence problem in the case of the Yolk but I am not aware of other clear mathematical result. The simulation results presented below give some clues.

\section{$3 \quad$ Voting rules and behavior}

I will be essentially interested in Plurality rule, the Borda rule, the Copeland rule and Approval Voting, when voters vote sincerely or strategically. Strategic behavior is introduced in a heuristic way as "responsive voting" without reference to equilibrium considerations.

\subsection{Sincere voting}

I consider three voting rules based on sincere behavior. The well-known Plurality rule, the Borda rule and the Copeland rule, which is a familiar Condorcet-consistent agregation rule. For Approval Voting, sincere voting (in the usual definition) does not provide a well-specified behavior. Behavior under this rule must be responsive and is thus described in the next section.

For a profile of strict preferences, the Plurality score of an alternative $k$ is simply the number of individuals whose best-prefered alternative is $k$. Plurality rule defines as winners the alternatives with largest plurality score. If preferences are not strict, the definition is naturally completed by saying that if $d$ distinct alternatives tie at the first rank in an individual preference ordering, then this individual gives, in the Plurality count, $1 / d$ point to each of them. If the individual preferences are not transitive, the Plurality rule has no straightforward extension and I shall not use it.

\footnotetext{
${ }^{3}$ Multidimensionnal Gaussian distributions are another option, used by Merill (1984) for simulations and by Laslier (2006) for the theory.
} 
Some pieces of notations will be useful. Recall that $m_{k, k^{\prime}}$ denotes the number of voter who (sincerly) prefer alternative $k$ to alternative $k^{\prime}$. The collum-sum of the matrix $M=\left(m_{k, k^{\prime}}\right)$ provides the candidates' Borda scores.

$$
b s(k)=\sum_{k^{\prime}=1}^{K} m_{k, k^{\prime}} .
$$

Replacing the matrix $M$ by the matrix $T=\left(t_{k, k^{\prime}}\right)$, with

$$
t_{k, k^{\prime}}=\left\{\begin{array}{cc}
1 & \text { if } m_{k, k^{\prime}}>n / 2 \\
0 & \text { if not }
\end{array}\right.
$$

one obtains the "tournament" matrix where $t_{k, k^{\prime}}=1$ means that a majority of voters prefers $k$ to $k^{\prime}$. The Copeland score $c s(k)$ of an alternative $k$ is the the number of other alternatives $k$ beats. It is easily computed from the tournament matrix.

$$
c s(k)=\sum_{k^{\prime}=1}^{K} t_{k, k^{\prime}}
$$

whose possible values ranges from 0 if $k$ is a Condorcet loser to $K-1$ if $k$ is a Condorcet winner. A Copeland winner is an alternative with maximal Copeland score. If there exists a Condorcet winner, this alternative is the unique Copeland winner.

\subsection{Responsive voting}

Here the voters respond to an annonced candidate score vector. The proposed reaction functions are derived from the theory of strategic voting: the voter holds some belief on the other voters' actions and rationally responds to this belief. The availlable information is essentially the same for all voters: it is a public signal about the popularity of the various candidate. In reality, such a signal is derived from the results of previous elections, from pre-electoral polls, or from any similar public information. Although different behaviors may appear as "rational" behavior within some fully specified game-theoretic models, the choices made here have the advantage of being comparable among different voting rules and cultures. In particular the public signal always takes the form of an announced ranking of the candidates. I do not need to suppose that a voter knows the other voters' preferences, or holds beleifs about them. In order to know what to do, a voter only has to figure out what the others do. 
The important point is that individual rational behavior cannot be defined, exept in general terms, knowing only the preferences. The answer to the question "Is it rational for me to cast this ballot" depends on what I beleive the other voters decide. It follows that a simulation approach to strategic voter behavior has to take the form of what is called here "responsive voting."

\subsubsection{Plurality voting}

Given announced scores for the various candidates, the voter votes for her preferred candidate among the two candidate with highest scores. If ties occur at the first places in the score vector, I introduce a small noise in the score vector to randomly break the ties and let the voter decide among two candidates only. Then, clearly, votes gather on two candidates only. If one of these two candidates is a Condorcet winner then this candidate wins, but it is possible that a Condorcet winner exists but votes nevertheless gather on other candidates. More exactly any candidate $k$ except a Condorcet loser can be elected, provided that people beleive that votes gather on $k$ and some other candidate $k^{\prime}$ which is losing in front of $k$ according to majority rule. On that point, see Cox (1997) and Myerson (2002) for the theory and Blais et al. (2008) for experiments. The theory, if not predictive, delivers a clear-cut message here, the path-dependence effect is so important that simulation work does not appear to be of interest. I will therefore not study strategic response in the case of Plurality voting. ${ }^{4}$

\subsubsection{Approval voting}

I use the strategic best-response function introduced and justified in Laslier (2009). Given approval scores for the various candidates (and if there are no ties in the first places) the voter considers the top-ranked candidate $k_{1}$, the "leader." She votes for or against the other candidates by comparing them to $k_{1}$. In order to decide whether she votes for or against the leader $k_{1}$ himself, she compares $k_{1}$ to $k_{2}$, the second-ranked candidate (the "challenger"). (See the example below.).

This response function has a fixed point if and only if there exists a Condorcet winner; in that sense, strategic approval voting is Condorcet-

\footnotetext{
${ }^{4}$ Lehtinen (2008) studies Plurality and Approval in some three-alternative societies, with voters' out-of-equilibrium beliefs based on perturbation of sincere voting vote shares. He concludes that Approval voting has a high utilitarian efficiency and Plurality has a low utilitarian efficiency, which is improved by strategic behavior. See also Lehtinen's contribution in this volume.
} 
consistent. If there is no Condorcet winner, the best response function is still well-defined, but the beliefs have to be specified. In the simulations I compute the first five iterations of this function, starting from a Condorcet-consistent sincere rule (I report on the Copeland rule, but using other Condorcet-consistent rules leads to the same conclusions). If there is a Condorcet winner, this candidate will not be defeated, and if there is no Condorcet winner, the procedure has no fixed points but looking at the first iterations may give a sense of what alternatives are selected by a society of strategic voters using approval voting.

\subsubsection{Borda voting}

I use the following response function, which is inspired from the previous one and could probably receive the same strategic justification. Given Borda scores $b s(k)$ of the various candidates $k$, and if there are no ties, the voter considers in turn $k_{1}, k_{2}, \ldots, k_{K}$ the candidates ordered according to the score vector $s$. First she compares the leader $k_{1}$ to his main challenger $k_{2}$. If she prefers $k_{1}$, she puts $k_{1}$ at the first place in her ballot and $k_{2}$ at the last place, thereby giving as many points as possible to $k_{1}$ and as few points as possible to $k_{2}$. If she prefers $k_{2}$ to $k_{1}$ she does just the contrary. Then she turns to $k_{3}$, the third-ranked candidate in $b s$. She only compares $k_{3}$ to $k_{1}$, because if there is to be a tie between two candidates involving $k_{3}$, it will most likely be a tie between $k_{3}$ and $k_{1}$. If she prefers $k_{3}$ to $k_{1}$,.she gives as many points as possible to $k_{3}$, and if she prefers $k_{1}$ to $k_{3}$, she gives $k_{3}$ as few points as possible. She will thus put $k_{3}$ in her ballot in the position 2 or $K-1$. Then she continues filling her Borda ballot this way until all the candidates have been compared with $k_{1}$.

Up to my knowledge, this heuristics has not been published for the study of strategic behavior under Borda rule. It amounts to suppose that the voter considers that the most likely ties are ordered by the score vector: $\left\{k_{1}, k_{2}\right\}$ is by far the most likely, followed by $\left\{k_{1}, k_{3}\right\},\left\{k_{1}, k_{4}\right\}$, etc.

\subsubsection{Example}

Suppose that there are five candidates $A, B, C, D, E$ and that the announced score vector ranks the candidates as follows:

$$
s(C)>s(A)>s(B)>s(D)>s(E)
$$

Consider an individual whose preference $P_{i}$ is:

$$
A P_{i} B P_{i} C P_{i} D P_{i} E
$$


- Under Plurality rule this individual will compare the leading candidates $C$ to his challenger $A$, and therefore vote " $A$ " because she prefers $A$ to $C$.

- Under Approval Voting, she will vote " $\{A, B\}$ " : The leader is $C$, thus $A$ and $B$ are approved because they are better than $C$, and $D$ and $E$ are not approved because they are worse than $C$. And $C$ himself is not approved because he is worse than the main challenger $(A)$.

- Under the Borda rule (with the scale $4,3,2,1,0$ ) she will give 4 points to $A, 0$ points to $C, 3$ points to $B, 1$ points to $D, 2$ points to $E$, thereby submitting the Borda-style ballot " $A \succ B \succ E \succ D \succ C$ ".

\subsubsection{Discussion}

The reactions function that I use are not the only possible ones but they have the advantage of being derived from the same idea, which is proeminent in the strategic voting literature: each voter considers that his vote is going to make a difference in the case of a tie between two candidates and responds individually to her subjective beliefs about the chances of the candidates by considering the likelyhood of possible ties.

I suppose that all voters simultaneously respond to the same belief, defined by a score vector. The interpretation is natural here in terms of preelection pools. This way of doing has the advantage that it makes possible to study strategic behavior out of equilibrium.

\section{Results}

I present (when possible) results for the winning alternative with sincere and responsive voting under Plurality, Borda, Copeland and Approval Voting. For responsive voting behavior, the winner may depends on the annonced ranking of candidates (the score vector) to which the voters react.

For Aproval Voting, I choose to look at the iterated reactions starting from the Copeland ranking of alternatives. This is because Aproval Voting, to many respect is a Condorcet-like voting method and Copeland is a Condorcet-consistent rule. For Borda, I naturally chose to iterate starting from the sincere Borda ranking. 


\begin{tabular}{|c|c|c|c|c|c|}
\hline$\left(\begin{array}{c}n=5 \\
K=5\end{array}\right)$ & $\beta=0$ & $\beta=.3$ & $\beta=.5$ & $\beta=.7$ & $\beta=1$ \\
\hline$\alpha=0$ & $.31(.07)$ & $.40(.20)$ & $.52(.30)$ & $.60(.36)$ & $.69(.42)$ \\
\hline$\alpha=.4$ & $.41(.22)$ & $.63(.43)$ & $.74(.52)$ & $.81(.58)$ & $.86(.62)$ \\
\hline$\alpha=.7$ & $.54(.40)$ & $.78(.61)$ & $.86(.68)$ & $.90(.72)$ & $.93(.75)$ \\
\hline$\alpha=1$ & $.70(.60)$ & $.87(.76)$ & $.92(.81)$ & $.96(.84)$ & $.98(.86)$ \\
\hline
\end{tabular}

Table 3: Rousseauist culture: probability of a Condorcet (a CondorcetRousseau) alternative for 5 voters and 5 alternatives

\begin{tabular}{|c|c|c|c|c|c|}
\hline$\left(\begin{array}{c}n=11 \\
K=5\end{array}\right)$ & $\beta=0$ & $\beta=.3$ & $\beta=.5$ & $\beta=.7$ & $\beta=1$ \\
\hline$\alpha=0$ & $.32(.06)$ & $.48(.29)$ & $.61(.39)$ & $.69(.44)$ & $.74(.48)$ \\
\hline$\alpha=.4$ & $.47(.33)$ & $.78(.62)$ & $.88(.69)$ & $.91(.72)$ & $.93(.74)$ \\
\hline$\alpha=.7$ & $.69(.61)$ & $.92(.81)$ & $.95(.85)$ & $.97(.86)$ & $.98(.87)$ \\
\hline$\alpha=1$ & $.87(.83)$ & $.98(.94)$ & $.99(.95)$ & $.99(.95)$ & $1.0(.95)$ \\
\hline
\end{tabular}

Table 4: Rousseauist culture: probability of a Condorcet (a CondorcetRousseau) alternative for 5 voters and 11 alternatives

\subsection{Results for Rousseauist cultures}

In this culture, Plurality voting is not well-defined in that case and I thus concentrate on the other rules. The first observation is that if the number of voters is large, because they are supposed to be independent, all voting rules detect the Rousseau winner with a high probability. I thus focus attention on small size societies (or "juries") and take $I=5$ and $I=11$.

Such a profile may have a Condorcet winner or not, but it is important to keep in mind that even if there exists a Condorcet winner, this alternative may different from the true best alternative, the "Rousseau" one. Table 3 and 4 show, for the case of $K=5$ alternatives and $n=5$ or 11 voters, the probability of the event "there exists a Condorcet winner" and, in brackets, the probability of the event "the Rousseau alternative is a Condorcet winner." These probabilities have been estimated from 1,000 draws of the above model. For instance, for $\alpha=.4$ and $\beta=.5$ (the values I will use later) and for $n=K=5$, in $74-52=22 \%$ of cases there is a Condorcet winner but this candidate is nevertheless the "wrong" one.

In order to evaluate voting rules and behaviors in such cultures, it is natural to observe the rank, according to the true ranking, of the chosen alternative. For Copeland rule and for Borda rule, this is well defined. For 


\begin{tabular}{|c|c|c|}
\hline$\alpha=.4, \beta=.5, K=5$ & $n=5$ & $n=11$ \\
\hline \hline Pr. of Condorcet : & .745 & .877 \\
\hline Pr. of Rousseau-Condorcet : & .518 & .693 \\
\hline \hline Rule & Average rank & Average rank \\
\hline \hline Copeland & 2.05 & 1.727 \\
\hline AV1 & 1.724 & 1.384 \\
\hline AV2 & 1.769 & 1.441 \\
\hline AV3 & 1.405 & 1.235 \\
\hline AV4 & 1.661 & 1.366 \\
\hline AV5 & 1.759 & 1.444 \\
\hline Borda & 1.268 & 1.116 \\
\hline Borda 1 & 2.586 & 2.832 \\
\hline Borda 2 & 1.557 & 1.342 \\
\hline Borda 3 & 2.140 & 2.155 \\
\hline Borda 4 & 1.605 & 1.403 \\
\hline Borda 5 & 1.901 & 1.654 \\
\hline
\end{tabular}

Table 5: Rousseauist culture: Average rank of the chosen alternative for 5 alternatives

responsive voting behavior, this depends on the score vector to which the voters react.

For Aproval Voting, I choose to look at the iterated reactions starting from the Copeland ranking of alternatives. This is because Aproval Voting, to many respect is a Condorcet-like voting method and Copeland is a Condorcet-consistent rule. For Borda, I naturally chose to iterate starting from sincere Borda ranking.

Some results are reported in Tables 5, and 6. These Tables report average ranks so, in reading them, one is interested in having ranks as small as possible.

Intuitively, in such culture, applying the Borda rule seems a better way to discover the best alternative than applying Condorcet-consistent choice rules, for the following reason.

If the randomly generated profile is very homogeneous and close to the true ranking, then all voting rules should agree. The difference between voting rules thus comes from the cases where enough mistakes have been done by the voters and, most importantly, from mistakes made when comparing the Rousseau winner to other alternatives. Because the probability of mistake decreases with the rank difference between alternatives, this probability 


\begin{tabular}{|c|c|}
\hline$\alpha=.4, \beta=.5, K=15$ & $n=11$ \\
\hline \hline Pr. of Condorcet : & .865 \\
\hline Pr. of Rousseau-Condorcet : & .670 \\
\hline \hline Rule & Average rank \\
\hline \hline Copeland & 2.35 \\
\hline AV1 & 1.41 \\
\hline AV2 & 1.47 \\
\hline AV3 3 & 1.24 \\
\hline AV4 & 1.40 \\
\hline AV5 & 1.46 \\
\hline Borda & 1.10 \\
\hline Borda 1 & 14.41 \\
\hline Borda 2 & 4.90 \\
\hline Borda 3 & 1.54 \\
\hline Borda 4 & 5.35 \\
\hline Borda 5 & 2.32 \\
\hline
\end{tabular}

Table 6: Rousseauist culture: Average rank of the chosen alternative for 15 alternatives

is the largest when comparing the Rousseau alternative to alternative number 2, the second-best one. It follows that the probability that the Rousseau alternative is detected as a Condorcet winner may be relatively low: as an extreme case (if $\alpha=0$ ) one may have that voters are wrong half of the time when comparing adjacent alternatives even if they are very skilled at comparing distant ones.

With the chosen parameters $(\alpha=.4, \beta=.5, n$ small) one obtains .518 , .693 and .670 in Tables 5, and 6. As a consequence, the performance of the Copeland method is rather poor: the average rank of the Copeland winner is $2.05,1.727$, and 2.35 .

The Borda rule also gathers information from other pairwise comparisons so that its performance is better than the performance of Copeland : The Borda winner has an average rank of $1.268,1.116$ and 1.10 with the same parameters.

From the simulations, we also learn that the Borda rule, in this favorable framework, behaves poorly with respect to manipulation, in contrast with Approval Voting.

The lines "Copeland, AV1, AV2, ..., AV5" depict successive strategic responses to the Copeland ranking, under Approval Voting. One can see 
that responsive voting is here beneficial.

The lines "Borda, Borda1,..." depict the successive strategic responses to the sincere Borda ranking. One can see that strategic behavior is here detrimental. This phenomena is very spectacular in the line "Borda 1" of Table 6 (the average rank is there 14.41 out of 15 candidates) and it can also be observed with a smaller number of candidates. Here is the explanation.

Consider the case of four alternatives, with true ranking

$$
1>2>3>4,
$$

and suppose first that all the voters agree on that ranking. Then sincere Borda obviously provides the true ranking. But then the strategic response of any voter is to rank the second alternative last, and to rank second the least dangerous alternative, that is the one with the lowest score, that is alternative 4, the worst one. This provides the (very un-sincere !) ballot:

$$
1>4>3>2 .
$$

Now suppose that a fraction $\varepsilon$ of the voters by mistake think that 2 is better than 1 . These voters tend to strategically rank 1 at the very last position, giving him as few points as possible in the Borda count, precisely because 1 is ranked first and appears thus as the most dangerous challenger for 2. Moreover those voters, just like the ones who made no mistake, will also put at the second position alternative 4, casting the ballot

$$
2>4>3>1 .
$$

The Borda score of alternative 1 (with the Borda scale 3, 2, 1, 0) is thus $(1-\varepsilon) \cdot 3+\varepsilon \cdot 0=3-3 \varepsilon$ and the Borda score of alternative 4 is $(1-\varepsilon) \cdot 2+\varepsilon \cdot 2=$ 2. It follows that, for $\varepsilon>1 / 3$, the alternative with highest Borda count is now alternative 4 , the worst one !

If there are only three alternatives, this phenomenon does not happen ${ }^{5}$, but if there are more alternatives, it becomes more frequent, even for small mistake probabilities. This situation occurs often in the simulation, which explains why the first-order strategic Borda winner is very badly ranked in these cultures.

One can see how strategic thinking with the Borda rule gives rise to erratic behavior, as reflected in these simulations. Of course this curious

\footnotetext{
${ }^{5}$ Many studies (Favardin et al. 2002, Myerson 2002, Lehtinen 2007) concentrate on three-alternative cases, and thus miss this effect.
} 


\begin{tabular}{|c|c|c|c|c|}
\hline$(1,000$ or 100 draws $)$ & $n=3$ & $n=5$ & $n=11$ & $n=99$ \\
\hline$K=3$ & .947 & .939 & .920 & .914 \\
\hline$K=5$ & .840 & .826 & .746 & .755 \\
\hline$K=15$ & .591 & .514 & .445 & .41 \\
\hline$K=50$ & .41 & .24 & .20 & \\
\hline
\end{tabular}

Table 7: Impartial culture: probability of a Condorcet winner

pattern is a consequence of the extreme assumption that all the voters react strategically and simultaneously to the same information. For instance the effect will be mitigated if some fraction of the voters vote sincerly by principle. Borda himself defended his method by saying that it is "intended for honest men." This is a lucid remark, but one should stress that, in the culture studied here, all voters share the same goal. Thus it is not clear that they should be labbelled as "dishonest" when trying individually to be as efficient as possible in reaching the common will, even if they end up in a collective faillure to do so.

\subsection{Results for impartial cultures}

Table 7 provides the frequency of the existence of a Condorcet winner, for some profil size. One can see that this probability decreases when the number of candidates grows. To compare voting schemes in the impartial culture, Table 8 indicates (for 11 voters and for 3,5 and 15 candidates) the probability for a given voting scheme, to elect the (sincere) Borda winner. ${ }^{6}$ For very small values of $K$, there is in general a Condorcet winner and, most often, this alternative is also the Plurality and the Borda winner and indeed the winner under most voting rules. So figures for $K=3$ are all very large. But when the number of individuals grows, things are quite diffrent. One can notice in particular that strategic voting makes the Borda prediction totally unstable: for $K=15$. Acoording to the Table, the probability that the strategic response to sincere Borda voting still elects the Borda winner is only $.163 .^{7}$

\footnotetext{
${ }^{6}$ Ties are broken randomly so that the Borda winner is always unique.

${ }^{7}$ Note that this has little to do with the probability that the Borda rule be manipulated, as ususally defined. Here all the voters vote responsively. Of course, if the number of individuals is not small, the probability that a single vote makes a difference is tiny.
} 


\begin{tabular}{|c|c|c|c|}
\hline$(n=11)$ & $K=3$ & $K=5$ & $K=15$ \\
\hline \hline Pr. Condorcet : & .920 & .746 & .445 \\
\hline \hline Rule & \multicolumn{3}{|c|}{ (Borda) } \\
\hline \hline Plurality & .800 & .586 & .248 \\
\hline \hline Copeland & .889 & .780 & .696 \\
\hline AV1 & .857 & .703 & .426 \\
\hline AV2 & .857 & .682 & .435 \\
\hline AV3 & .889 & .770 & .569 \\
\hline AV4 & .857 & .721 & .491 \\
\hline AV5 & .857 & .686 & .447 \\
\hline \hline Borda & 1 & 1 & 1 \\
\hline Borda 1 & .863 & .514 & .163 \\
\hline Borda 2 & .846 & .690 & .545 \\
\hline Borda 3 & .879 & .547 & .388 \\
\hline Borda 4 & .860 & .698 & .464 \\
\hline Borda 5 & .852 & .690 & .489 \\
\hline
\end{tabular}

Table 8: Impartial culture with 11 voters: Probability of chosing the Borda winner

\begin{tabular}{|c|c|c|c|c|}
\hline$(1,000$ or 100 draws $)$ & $n=3$ & $n=5$ & $n=11$ & $n=99$ \\
\hline$K=3$ & .751 & .790 & .752 & .761 \\
\hline$K=5$ & .379 & .348 & .351 & .39 \\
\hline$K=15$ & .004 & .005 & .002 & .00 \\
\hline
\end{tabular}

Table 9: Consensual distributive culture: probability of a Condorcet winner

\subsection{Results for consensual distributive cultures}

Table 9 provides the frequency of the existence of a Condorcet winner, for various profil sizes. One can see that this probability tends quickly to 0 when the number of candidates grows.

A specific feature of this culture is that the Gini index of inequality tends to $1 / 3$ when the number $n$ of voters tends to infinity. For instance, for $n=11$, the expected value of this index is .31 . This is what would be obtained on average if there was no vote but a random choice. Table 10 provides the average values of the Gini index for the winning alternative according to different voting schemes. Note that the three value $K=3,5,15$ chosen for the number of candidates give rise to preference profiles which are 
quite different one from the other since the frequency of Condorcet winners goes from $75 \%$ to $2 \%$. In terms of inequality, one notices that Plurality voting does slightly worse than a random choice whereas the other schemes do slightly better.

The interpretation of these results must be related to the shape of the probability distribution over the set of alternatives that this culture defines. The main question that is raised when comparing voting rules in redistributive settings is to know to what extend a voting schemes tends to select more or less egalitarian alternatives. But in the consensual distributive culture, the probability distribution over the set of alternatives is such that existing alternatives tend to be similar to that respect. It is therefore delicate to disentangle by simulation this effect from the effect of the different voting rules. Since there is no reason to believe that the consensual distributive culture is close to any "real" culture (despite its mathematical simplicity), I conclude that one should rather use a different approach in order to study by simulation the redistribution problem. This is why I introduced the other kind of distributive cultures, called "inegalitarian distributive cultures," which I will study now.

\subsection{Results for inegalitarian distributive cultures}

For the simulations I chose the parameters

$$
\begin{aligned}
e_{\min } & =1.3 \\
e_{\max } & =4 .
\end{aligned}
$$

With this values, the interval of possible values for the Gini index of inequalities roughly covers the actual national values of this coefficient for income distributions. But the Gini formula is not linear with the parameter $e$. With the uniform distribution for the parameter $e$ on the interval $\left[e_{\min }, e_{\max }\right]$, one obtains a probability distribution for the Gini index as depicted in Figure 2.

Table 11 provides the probability of the existence of a Condorcet winner, for various profile sizes. One can see that this probability varies a lot with the number of candidates and, perhaps more surprisingly, the number of individuals.

Voting rules and behaviors are compared with respect to the degree of inequality proposed by the winning candidate, measured by the Gini index. Table 12 shows such results for the case of $n=11$ individuals (or 11 groups of individuals of equal size). One can see that Plurality rule behaves very poorly, indeed worse than the mere random choice of an alternative. 


\begin{tabular}{|c|c|c|c|}
\hline$(n=11)$ & $K=3$ & $K=5$ & $K=15$ \\
\hline \hline Pr. Condorcet : & .752 & .351 & .002 \\
\hline \hline Rule & \multicolumn{3}{|c|}{ Gini } \\
\hline \hline random choice & .31 & .31 & .31 \\
\hline \hline Plurality & .32 & .36 & .40 \\
\hline \hline Copeland & .30 & .30 & .29 \\
\hline AV1 & .31 & .30 & .31 \\
\hline AV2 & .31 & .31 & .31 \\
\hline AV3 & .30 & .30 & .31 \\
\hline AV4 & .31 & .31 & .30 \\
\hline AV5 & .31 & .31 & .30 \\
\hline \hline Borda & .31 & .30 & .29 \\
\hline Borda 1 & .31 & .31 & .31 \\
\hline Borda 2 & .31 & .31 & .31 \\
\hline Borda 3 &. .31 & .31 & .31 \\
\hline Borda 4 & .31 & .30 & .30 \\
\hline Borda 5 & .31 & .31 & .31 \\
\hline
\end{tabular}

Table 10: Consensual redistributive culture with 11 voters: Average inequality of the chosen alternative

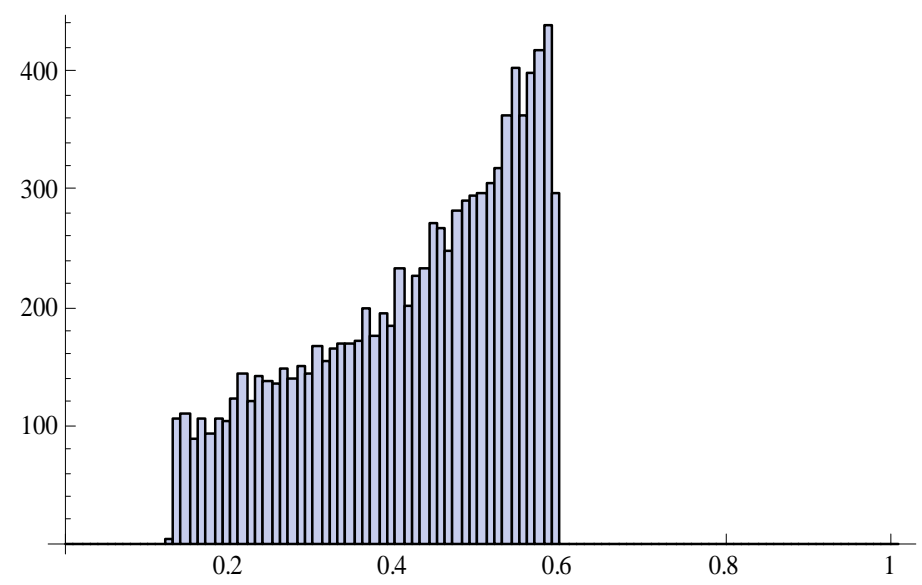

Figure 2: Inegalitarian distributive culture: histogram for the Gini index 


\begin{tabular}{|c|c|c|c|c|}
\hline$(1,000$ or 100 draws $)$ & $n=3$ & $n=5$ & $n=11$ & $n=99$ \\
\hline$K=3$ & .803 & .800 & .825 & .941 \\
\hline$K=5$ & .419 & .403 & .500 & .81 \\
\hline$K=15$ & .013 & .013 & .029 & .21 \\
\hline$K=50$ & .00 & .00 & & \\
\hline
\end{tabular}

Table 11: Inegalitarian distributive culture: probability of a Condorcet winner

This is an interesting observation, and an argument against Plurality voting with three or more candidates. The phenomenon is even spectacular when the number of voters and candidates is large. For instance with $n=99$ voters and $K=15$ candidates, the plurality winners has an average Gini index of .57, whereas a random choice yields .42. The intuition is that in order to be the preferred alternative of several voters, a candidate must propose to each of them more than what is proposed by the other candidates. In order to do so, the candidate should propose very little to the voters which are not targeted, hereby proposing a relatively unequal distribution. Following this mechanism, in this setting, Plurality rule promotes inequality.

Other voting rules, such as Copeland and Borda do not suffer this pathology and designate alternatives with smaller Gini index. Voter strategic behavior in that case is detrimental to equality, although not as detrimental as sincere Plurality.

\subsection{Results for spatial cultures}

\subsubsection{Results for unidimensional culture}

Results for the unidimensional case are reported in Table 13 for $n=11$ voters. This Table indicates for the various rules how frequent is the election of the Condorcet winner. Since a Condorcet winner always exists in this culture, the Copeland rule and the Approval Voting responses to the Copeland ranking always elect the Condorcet winner.

Plurality does not often elects the Condorcet winner. Note that, since positions are chosen at random and uniformly, up to some border effects, all candidates are equally likely to be chosen by Plurality rule.

The Borda rule does much better under sincere voting than Plurality. The iterated strategic responses to the Borda ranking do very bad in the first iteration, as was already seen in previous sections but the situation here improves with successive iterations. 


\begin{tabular}{|c|c|c|c|}
\hline$(n=11)$ & $K=3$ & $K=5$ & $K=15$ \\
\hline \hline Pr. Condorcet $:$ & .825 & .500 & .029 \\
\hline \hline Rule & \multicolumn{3}{|c|}{ Gini } \\
\hline \hline random choice & .42 & .42 & .42 \\
\hline \hline Plurality & .42 & .47 & .54 \\
\hline \hline Copeland & .36 & .32 & .26 \\
\hline AV1 & .36 & .35 & .35 \\
\hline AV2 & .36 & .35 & .37 \\
\hline AV3 & .36 & .34 & .36 \\
\hline AV4 & .36 & .34 & .36 \\
\hline AV5 & .36 & .35 & .35 \\
\hline \hline Borda & .36 & .32 & .26 \\
\hline Borda 1 & .36 & .39 & .40 \\
\hline Borda 2 & .36 & .35 & .36 \\
\hline Borda 3 & .36 & .37 & .36 \\
\hline Borda 4 & .36 & .35 & .36 \\
\hline Borda 5 & .36 & .36 & .35 \\
\hline
\end{tabular}

Table 12: Inegalitarian redistributive culture with 11 voters: Average inequality of the chosen alternative 


\begin{tabular}{|c|c|c|c|}
\hline$(n=11)$ & $K=3$ & $K=5$ & $K=15$ \\
\hline \hline Pr. Condorcet : & 1 & 1 & 1 \\
\hline \hline Rule & \multicolumn{3}{|c|}{ (Condorcet) } \\
\hline \hline Plurality & .736 & .528 & .305 \\
\hline \hline Copeland & 1 & 1 & 1 \\
\hline AV1 & 1 & 1 & 1 \\
\hline AV2 & 1 & 1 & 1 \\
\hline AV3 & 1 & 1 & 1 \\
\hline AV4 & 1 & 1 & 1 \\
\hline AV5 & 1 & 1 & 1 \\
\hline \hline Borda & .868 & .777 & .616 \\
\hline Borda 1 & .850 & .508 & .158 \\
\hline Borda 2 & .996 & .557 & .166 \\
\hline Borda 3 & .983 & .732 & .223 \\
\hline Borda 4 & .999 & .739 & .271 \\
\hline Borda 5 & .998 & .806 & .340 \\
\hline
\end{tabular}

Table 13: Uni-dimensional culture with 11 voters: Probability of chosing the Condocet winner

In spatial cultures, it makes sense to consider large numbers of voters. Further exploration in this direction, which are not reported here, confirm the above findings.

\subsubsection{Results for multi-dimensional culture}

Results for a two-dimensional case are reported in Table 14 for $n=11$ voters. Bliss points and alternatives are drawn from a rectangle of size $1 \times .5$. In this cultures, it is possible that no Condorcet winner exist, but this is a rare phenomenon if the number of alternatives is not large. I therefore take as a reference point a Condorcet-consistent voting scheme: the Copeland rule. This Table indicates for the various rules how frequent is the election of the Copeland winner. Since a Condorcet winner often exists in this culture, the Copeland rule and the Approval Voting responses to the Copeland ranking usually coincide (and elect the Condorcet winner).

Plurality behaves differently Note that, since I choose positions at random and uniformly, up to some border effects, all candidates are equally likely to be chosen by Plurality rule.

The Borda rule does much better under sincere voting than Plurality. 


\begin{tabular}{|c|c|c|c|}
\hline$(n=11)$ & $K=3$ & $K=5$ & $K=15$ \\
\hline \hline Pr. Condorcet : & .989 & .966 & .833 \\
\hline \hline Rule & \multicolumn{3}{|c|}{ (Copeland) } \\
\hline \hline Plurality & .782 & .538 & .233 \\
\hline \hline Copeland & 1 & 1 & 1 \\
\hline AV1 & .989 & .966 & .833 \\
\hline AV2 & .989 & .966 & .833 \\
\hline AV3 & .989 & .998 & .935 \\
\hline AV4 & .989 & .967 & .860 \\
\hline AV5 & .989 & .966 & .841 \\
\hline \hline Borda & .874 & .538 & .624 \\
\hline Borda 1 & .861 &. .479 & .122 \\
\hline Borda 2 & .988 & .500 & .133 \\
\hline Borda 3 & .966 & .661 & .316 \\
\hline Borda 4 & .990 & .674 & .393 \\
\hline Borda 5 & .965 & .753 & .476 \\
\hline
\end{tabular}

Table 14: Bi-dimensional rectangular culture with 11 voters: Probability of chosing the Condocet winner

The iterated strategic responses to the Borda ranking do very bad in the first iteration, as was already seen in previous sections when the number of candidates is not very small but the situation here improves with successive iterations.

In spatial cultures, it makes sense to consider large numbers of voters. Further exploration in this direction confirm the above findings One point should neverthless be stressed. With uniform probability distributions on boxes, drawing a large number of points tends to produce ever more symmetric patterns. Empirical distributions tend to ressemble the uniform continous distribution, which is a very specific situation (mistakenly considered as "general" by Tullock (1967)). For instance, here are some results obtained in the four-dimensionnal culture on the box $1 \times 1 \times 1 \times 1$. With $K=3$ alternatives and $n=5$ voters a Condorcet winner exists most often (observed frequency: 97\%). Increasing the number of alternatives makes this freaquency decrease, in conformity with the "chaos" ideas of Spatial Voting theory. With $K=50$ alternatives, the observed frequency is $46 \%$. But picking uniformly many voters makes this frequency increase. With $K=50$ alternatives and $n=99$ voters, the observed frequency is $91 \%$. 


\section{Conclusion}

This study confirms what has been observed theoretically and empirically: (1) Voting rules exist that improve substancially on Plurality rule. (2) Apart the voting rule itself, the behavior of voters is of primary importance to predict the outcome of an election and therefore to assess the quality of a voting rule.

One point that is emphasised by these simulations is that the way we should judge voting rules depends also on the context. What is the number of voters and options? What are these options ? Are we dealing with a problem of shared interest or a conflict? All these questions are relevant.

With respect to strategic voting it appears that the use of the Borda rule may generate substancial perverse effects, in particular if there are many alternatives on the agenda. Comparatively, Approval voting does not seem to generate such pathologies. This may be related to the fact that strategic behavior under Approval Voting is usually sincere (in fact in the model which was used, such is always the case) according to the usual definition of sincerity for Approval Voting ("If you approve A and not B then you prefer A to B"). On the contrary, strategic behavior under the Borda rule may produce very un-sincere votes. 


\section{A Appendix}

\section{A.1 Standard deviation in the consensual distributive cul- ture}

The variables $y_{i}, i=1, \ldots, n$ are independent and uniform on $[0,1]$. Let $s=\sum_{i=1}^{n} y_{i}$ and $x_{i}=y_{i} / s$. The standard deviation $d(x)$ of $x$ is such that

$$
\begin{aligned}
d(x)^{2} & =\frac{1}{n} \sum_{i}\left(\frac{y_{i}}{s}-\frac{1}{n}\right)^{2} \\
& =\frac{1}{s^{2}} \frac{1}{n} \sum_{i}\left(y_{i}-\frac{s}{n}\right)^{2}
\end{aligned}
$$

When $n$ tends to infinity, the sum $\frac{1}{n} \sum_{i}\left(y_{i}-\frac{s}{n}\right)^{2}$ tends to the theoretical variance of the uniform distribution on $[0,1]$, that is a fixed positive number. Because, $s / n$ tends to the expected value $1 / 2$, one can see that $d(x)$ tends to 0 as $n^{-1}$.

\section{A.2 Gini index in the consensual distributive culture}

The index is:

$$
\text { gini }=\frac{2}{n} \sum_{i=1}^{n}\left(\frac{i}{n}-v_{i}\right)
$$

where $v_{i}$ denotes the (partial ordered) sum of the $i$ smallest values of the $n$ variables described above. One can write

$$
\text { gini }=\frac{2}{n} \sum_{i=1}^{n}\left(\frac{i}{n}-\sum_{j=1}^{i} \frac{u_{i}}{s}\right) .
$$

For $n$ large, $s$ is close to $n / 2$ so that gini is close to

$$
\frac{2}{n^{2}} \sum_{i=1}^{n} i-\frac{4}{n^{2}} \sum_{i=1}^{n} \sum_{j=1}^{i} u_{i}
$$

The first term tends to 1 . For the second term, note that $\sum_{j=1}^{i} u_{i} \simeq i^{2} /(2 n)$ and $\sum_{i=1}^{n} i^{2} \simeq i^{3} / 3$. Thus the second term tends to $2 / 3$ and one can see why gini tends to $1 / 3$. 


\section{References}

[1] David Austen-Smith and Jeffrey S. Banks (1999) Positive Political Theory 1: Collective Preferences. Ann Arbor: University of Michigan Press.

[2] André Blais, Jean-François Laslier, Nicolas Sauger, and Karine Van der Straeten (2008) "Sincere, strategic, and heuristic voting under four election rules: an experimental study" mimeo.

[3] Condorcet (1785) Essai sur l'application de l'analyse à la probabilité des décisions rendues à la pluralité des voix. Paris: Imprimerie Royale.

[4] Gary W. Cox (1997) Making Votes Count: Strategic Coordination in the World's Electoral Systems Cambridge: Cambridge University Press.

[5] Pierre Favardin, Dominique Lepelley, and Jerôme Serais, "Borda rule, Copeland method and strategic manipulation" Review of Economic Design 7: 213-228.

[6] William V. Gehrlein and Peter C. Fishburn (1979) "Proportions of profiles with a majority candidate" Computers and Mathematics. with Applications. 5:117-124.

[7] William V. Gehrlein (1997) "Condorcet's paradox and the Condorcet efficiency of voting rules" Mathematica Japonica 45:173-199.

[8] Jean-François Laslier (2002) "How two-party competition treats minorities" Review of Economic Design 7: 297-307.

[9] Jean-François Laslier and Nathalie Picard (2002) "Distributive politics and electoral competition" Journal of Economic Theory 103:106-130.

[10] Jean-François Laslier (2006) "Spatial approval voting" Political Analysis 14: $160-185$.

[11] Jean-François Laslier (2009) "The Leader Rule: A model of strategic approval voting in a large electorate" Journal of Theoretical Politics 21: 113-136.

[12] Aki Lehtinen (2007) "The Borda rule is also intended for dishonest men" Public Choice 133: 73-90.

[13] Aki Lehtinen (2008) "The welfare consequences of strategic behavior under approval and plurality voting" European Journal of Political Economy 24: 688-704. 
[14] Assar Lindbeck and Jörgen Weibull (1987) "Balanced-budget redistribution as the outcome of political competition" Public Choice 52: 273-297.

[15] Alessandro Lizzeri (1999) "Budget deficit and redistributive politics" Review of Economic Studies 66: 909-928.

[16] Richard D. McKelvey (1986) "Covering, dominance, and institutionfree properties of social choice" American Journal of Political Science 30: 283-314.

[17] Richard D. McKelvey and Craig A. Tovey (2008) "Aproximation of the Yolk by the LP Yolk" Mathematical Social Sciences, forthcoming.

[18] Samuel Merrill, III (1984) "A comparison of efficiency of multicandidate electoral systems" American Journal of Political Science 28: 23-48.

[19] Roger B. Myerson (1993) "Incentives to cultivate favored minorities under alternative electoral systems" American Political Science Review 87: 856-869.

[20] Roger B. Myerson (2002) "Comparison of scoring rules in Poisson voting games" Journal of Economic Theory 103: 219-251.

[21] Hannu Nurmi and Hannu Salonen (2008) "More Borda Count Variations for Project Assessment" AUCO Czech Economic Review 2: 109-22.

[22] Jean-Jacques Rousseau (1762) Du contrat social, Amsterdam: MarcMichel Rey.

[23] Michel Truchon (2008) "Borda and the Maximum Likelihood Approach to Vote Aggregation" Mathematical Social Sciences 55: 96-102.

[24] Michel Truchon and Mohamed Drissi-Bakhkhat (2004) "Maximum Likelihood Approach to Vote Aggregation with Variable Probabilities" Social Choice and Welfare 23: 161-185.

[25] Gordon Tullock (1967) "The general irrelevance of the general impossibility theorem" The Quarterly Journal of Economics 81: 256-270.

[26] H. Peyton Young (1988) "Condorcet's theory of voting" American Political Science Review 82: 1231-1244. 